\title{
Using a crowdsourcing knowledge base to support the Sustainability and Social Compromise skill in Computer Science Engineering Studies
}

\author{
Marc Alier Forment ${ }^{1}$, David López ${ }^{2}$, Fermin Sánchez Carracedo ${ }^{2}$, Jordi Garcia \\ Almiñana ${ }^{2}$, Jordi Piguillem Poch ${ }^{1,}$ and Martha Velasco ${ }^{2}$ \\ ${ }^{1}$ Universitat Politècnica de Catalunya, C/Jordi Girona Salgado 1-3, Office Omega 116 \\ 08034 Barcelona, Spain \\ ludo@essi.upc.edu, jpiguillem@essi.upc.edu \\ ${ }^{2}$ Universitat Politècnica de Catalunya, C/Jordi Girona Salgado 1-3, Building D6 \\ 08034 Barcelona, Spain \\ david@ac.upc.edu, fermin@ac.edu , jordig@ac.upc.edu
}

\begin{abstract}
The Skill "Sustainability and Socia Commitment" is commonly accepted as essential in today's world. However it proves tricky to introduce into the curriculum, mainly because of lack of knowledgeable teachers. To address this issue we present a knowledge base that brings together scientific articles, books, videos, compilations of data, experiences, etc., related to sustainability and knowledge areas associated with computer science engineering. This is a valuable tool that should provide to the teacher accurate and useful information in the research task of finding links between her course and the "Sustainability and Social Commitment" skill.
\end{abstract}

Keywords: Education, Skills, Sustainability, Educational resources, knowledge society

\section{Introduction}

The adaptation to the European Higher Education Area (EHEA) has brought us the inclusion of professional skills in the curricula of universities. There are many studies on the need to incorporate these skills $[1,4,9,10]$. The need of most of these skills is not discussed while others have fewer consensuses. There are also skills with which the teachers feel more comfortable, because they have an idea of how to teach and evaluate them.

The professional skill "Sustainability and Social Commitment" (from now on SSC) is generally accepted as essential in the modern world (despite some detractors), but raises concerns between teachers due to the lack of knowledge about it. It is very common, when discussing the skill, to hear questions such as: What is sustainability? 
What is its relationship with my subject? What should my students learn? How to evaluate the skill?

There exist several studies on the overall relationship between computers and SSC $[6,7]$, how to include this skill in some subjects $[3,5,8,10]$, in a whole curriculum [11] or how to evaluate it $[2,5,7,8]$. However, these studies do not solve a complex problem: that every teacher knows how it relates SSC competition with his subject.

In most schools, it is not a hard work to find a group of teachers who are interested in SSC. But once you have the group of teachers, they cannot be left alone, without supporting them, telling them to look for themselves the relationship between their area of knowledge and SSC, and asking them to develop new activities from scratch. If there is not some support, the initiative will fail.

Therefore, our University (UPC - Barcelona Tech) has developed the project STEP (Sustainability, Technology and Excellence Program). This project provided financial support to groups of teachers of every school at the University, to develop the tools, activities and ideas needed to introduce the SSC skill in the new degrees adapted to EHEA. The authors of this paper are the responsible of the STEP project at the Barcelona School of Informatics, and we must help to introduce the SSC skill in the new Degree in Computer Science. Among other activities, described in [9] we have developed a tool to support teachers interested in working in the skill: SSC Knowledge Base.

This tool brings together a selected group of articles in scientific and popular journals, databases related to SSC, pages of corporate social responsibility from software and hardware companies, a compendium of laws, directives, recommendations, good practice examples and activities to do in class, etc. All these inputs are oriented to relate SSC concepts with the typical knowledge areas of Computer Science.

All entries have a small comment made by the person who entered the data and a series of tags, the keywords associated with each entry that allow us to perform very specific searches.

The advantages of this tool versus a generic search engine such as Google, are:

- The information is bounded. With certain keywords, Google can go from hundreds to millions of responses, most of which are not relevant to the person searching. In our case, all entries have been shortlisted, so that the number of results will be much smaller, and the probability of finding the information you are searching for is greater.

- The information has been introduced with some comments. These comments can be from a couple of lines with a basic review, to a summary of the article or more elaborate information. These comments can offer a first look of the contents of the entry without access to it.

- The information is tagged, so the person who entered the data have read (albeit briefly) the content of the entry, and decided that the entry should be indexed under certain tags, which allows you to search quite efficient and accurate. The user can also search for words outside the list of tags, just on the title and comment from every input of the Knowledge Base. 
With this tool, teachers can find items that relate his subject with the SSC skill, so they can search for activities, finding specific examples or just real information to design their own activities and exercises, all quickly and easily.

The rest of the paper is organized as follows: Section 2 presents the process of discussion that led to the organization of the Knowledge Base and tagging system. Section 3 presents the technical specifications of the tool. Section 4 presents an example of use. Finally, Section 5 presents some final thoughts and conclusions.

\section{The process of definition of the Knowledge Base}

To create the knowledge base was necessary to design initially its structure and functionality. We looked for a knowledge base which locates entries based on keywords, but in a very open way (almost any word you could imagine). Moreover, we wanted the association of keywords to entries was easy and comfortable to carry and update entries. We needed, therefore, a small set of keywords associated as labels to the entries, but we also wanted the search could be made by using a much higher keyword set. To allow for complex searches, a certain structure on keywords was required. After analyzing different possibilities, we decided to provide the knowledge base of a three-dimensional structure.

On the one hand, we have a two-dimensional structure of keywords: a main level and secondary level. We defined a minimal yet complete set of keywords on the main level to cover all aspect. These words have a very high level of abstraction. The secondary level consists of a short but comprehensive set of labels covering more specific concepts. A direct relationship exists between keywords on the main and secondary level. The same word at the secondary level can be related to one or more keywords in the main level. This multiple assignment allows the possibility of complex searches. For example, searching for entries related to the secondary keyword $\mathrm{X}$ you can find all entries labeled directly with $\mathrm{X}$ or, in addition, those associated with the main keywords related also to the mark X (all the word with an $X$ in the column in Figure 1).

Keywords defined on the main level are the following:

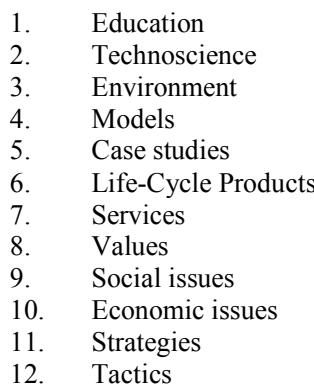

Figure 1 shows the relationship between keywords from main and secondary level. The first column shows the list of keywords from the secondary level. Remaining columns indicate, with an X, when a word from the secondary level is directly related to one from the main level. As can be seen, the main level consists of words with a level of abstraction higher than the words from secondary level. 


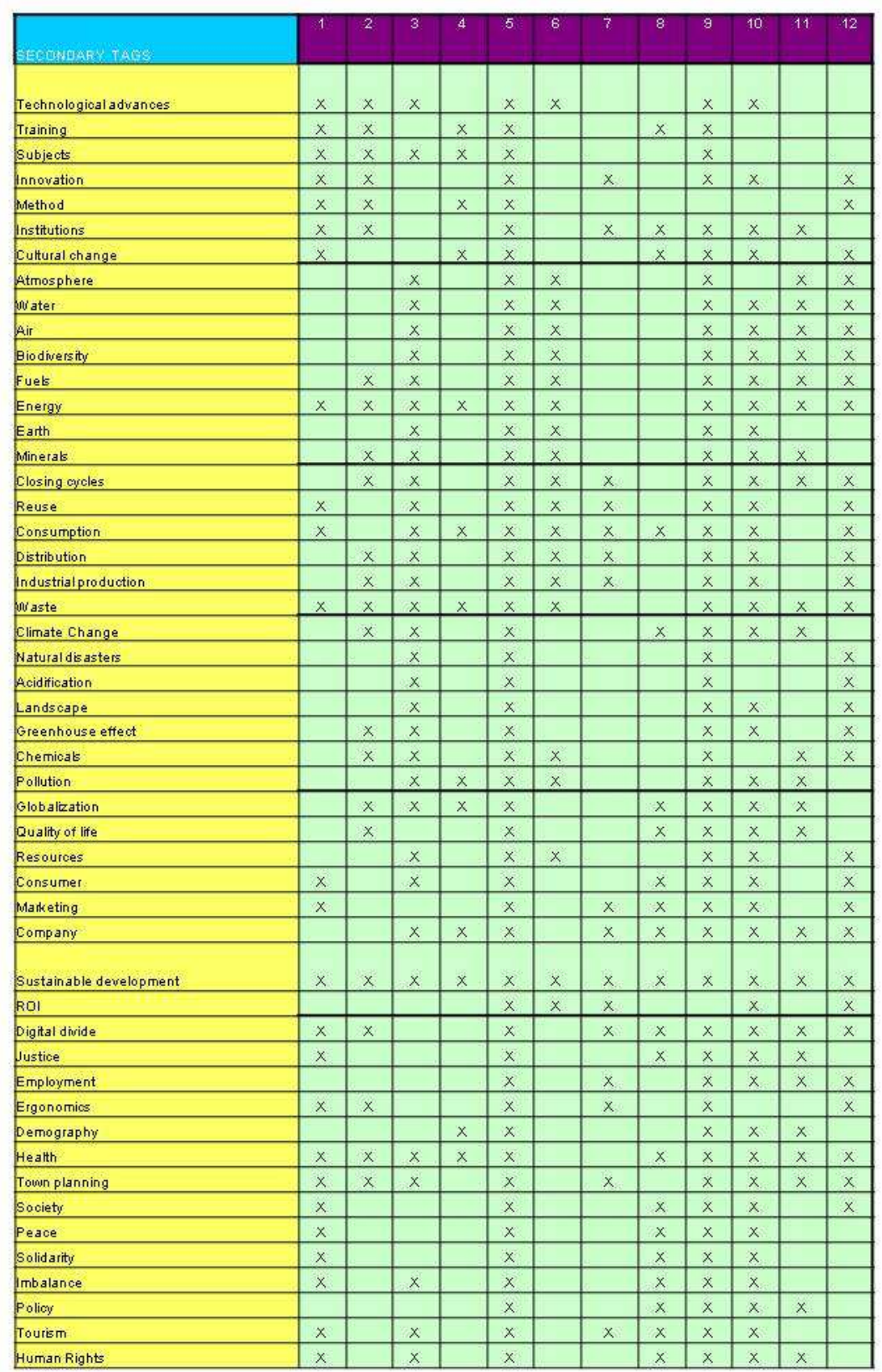

Figure 1. Relationship between keywords from the main and secondary levels 
We spent four months in selecting keywords, both the main level and secondary level, meeting us weekly during this time. In the meantime between meetings we classified sites, papers and other entries by using the keywords we had selected, and provisionally we included new keywords when necessary. The definitive addition to the knowledge base was made in our weekly meeting, and always in agreement of all of us. Once all keywords were selected, we established the relationship between main and secondary level. To this end, each of us made his own assignment, which after was reviewed by all together. For those assignments we didn't agree, we performed a new individual assignment, after discussing each keyword, and we established those assignments that had majority agreement.

On the other hand, in parallel with the process above described, we define the third dimension of the table. To enable searching for entries from a large number of different keywords, we define for each keyword at the secondary level a set of synonyms or related words. Entries in the knowledge base can also be found by using this "synonymous", despite being labeled only by keywords from secondary level. For example, for the keyword "biodiversity" we defined as synonymous the following words: "Fauna, Flora, Species, Biosphere, Nature, Extinction, Animals, Plants, Forest, Trees, Hunting and Fishing." Thus, an entry could be labeled as biodiversity but also appeared when searching for any of these related words.

The existence of "related words" allows us to easily update the knowledge base and adding new keywords without having to review all entries, just adding the new keywords as related words. This third dimension has helped further the multilingual capabilities of the knowledge base, because related words can also be translations to other languages of all the words from the list. By orthogonal, we defined a list of related words also for each keyword on the main level.

The entire process of creating the structure of keywords, starting from the first discussions on its structure until the final definition of the words in each level and their related words, lasted over six months. This process, in addition to the undersigned authors, involved two fellows who are primarily responsible for maintaining the list and structure of keywords and catalog entries from pre-defined keywords. One of the fellows had extensive training on issues related to SSC (we select her right after she read her $\mathrm{PhD}$ thesis, concerning this topic), while the other was a non-graduate student of computer engineering. In the implementation of the knowledge base a postgraduate student was also been involved.

\section{Specifications and technical details of the implementation of the Knowledge Base}

Prior to the implementation phase, there was a gathering of requirements for the Knowledge based that can be summarized as the following:

- It needed to be an online system, based on the web to maximize the ease of access, usability and for it to be cross platform.

- It needed to implement a documental database able to refer to different kinds of contents. 
- It needed to implement a tagging engine, so the taxonomy described in section 2 could be implemented, and support search.

- It needed to allow access control with several different levels of granularity.

- It needed to keep access logs, so statistics and data-mining practices on its use could be performed

- It needed to be a really simple environment, with a fair learning curve; and if possible already familiar for the collective of professors and lecturers.

To use a relational database or a custom development where discarded in the first round of analysis, due to cost and time constrains, and also because the risk it would imply such project. It was seriously considered to use a Wiki engine, like the software Mediawiki (mediawiki.org) in which is based the Wikipedia (Wikipedia.org) Wikis are very powerful documental databases, collaboratively created, that allow virtually any kind of structure and navigation scheme. But as a drawback, wiki projects require a continuous attention and content curation; otherwise there is a good chance that the structure and information quality will degrade with the use. The complexity of the Mediawiki source code and other wiki engines that where considered for the project caused, at last the wiki option to be discarded.

A strong alternative was to use a service of Social Bookmarking, based on freemium software as a service online tools like http://Delicius.com and http://Diigo.com. These services allow the creation of collections of references to URIs (Universal Location Identifiers) tagged according to flexible taxonomies (defined organically) as the groups of users work on the knowledge base. Social Bookmarking services added a nice to have feature: each user can create and maintain its own list of references and re-tag it according to her own criteria. References could be shared among a group of users, adding a -so to speak- 2.0 flavor to the Knowledga base.

As a critical drawback to use Social Bookmarking services implies to give away the control of the Knowledge Base to an external company, located in another country, that provides a free service with a service agreement that do not gives guaranties of any kind that the Knowledge Base and service might prevail over time, nor gives any kind of guaranty in case of loss on information. While there is the option of getting a backup of one user Bookmarks, is not possible to get the whole Knowledge Base baceuse it would belong to several users. Thus a decision was taken to look for a software that would implement the features of a Social Bookmarkin that we could set up on the servers in our center.

On 2008 Jordi Piguillem Poch, PhD student of the ESSI department of the UPC and core developer of Moodle (http://moodle.org), developed a Moodle contrib module that implements a course activity that implements a social Bookmarking within the course. Moodle is the same LMS that implements the UPC and the vast majority of the spanish universities. For the academic personal Moodle a well known system, it also allows a very granular access control and keeps a detailed access log.

In a couple of weeks of work, Piguillem adapted the initial design of his module to support the specific requirements defined by the taxonomy described in section 2 . The final implementation of the Knowledge base is based on Moodle 1.9.11 with a modified version of the Open Sourced Module that can be found at http://code.google.com/p/moodle-social-bookmarking/ 


\section{Example of use}

The Knowledge Database described in this work is publicly accessible to any user through the web address http://sycs.fib.upc.edu/ and its use is very simple. After an initial presentation screen of the knowledge database, the user is routed directly to the search screen.

Figure 2 shows a screenshot of the screen, the search one. As you can see this screen contains, in the center, the text box to start a search, and a cloud box with information to the list of predefined tags.

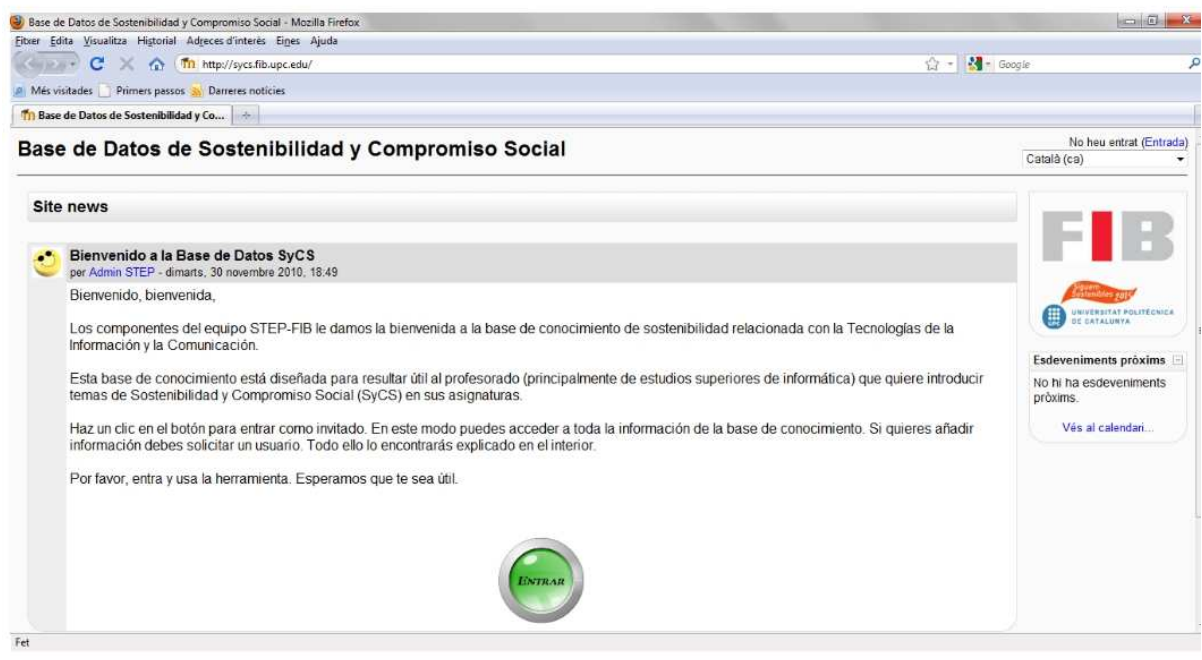

Figure 2. Main screen.

A search can be done in two ways:

- Using a predefined label with which all entries have been classified, or

- Using free text to be searched among the title and the description of the entry.

In order to search through a predefined label, the label can directly be selected from the right cloud box, or it can be written in the text box preceded by the mark "tag". For example, to search for entries tagged as solidarity, you can write directly: "tag: solidarity". 
If you want to search using free text, you can type the text directly into the box. For example, typing United Nations all entries containing these words in the title or description fields will be displayed.

In Figure 3 you can see a screenshot example of the search results by "tag: operating systems". You can see a list of selected entries that contains the title, description, and the list of labels that classify that input. The title, in turn, is a link to the resource, either a web page, an article (usually in PDF format), a video, etc.

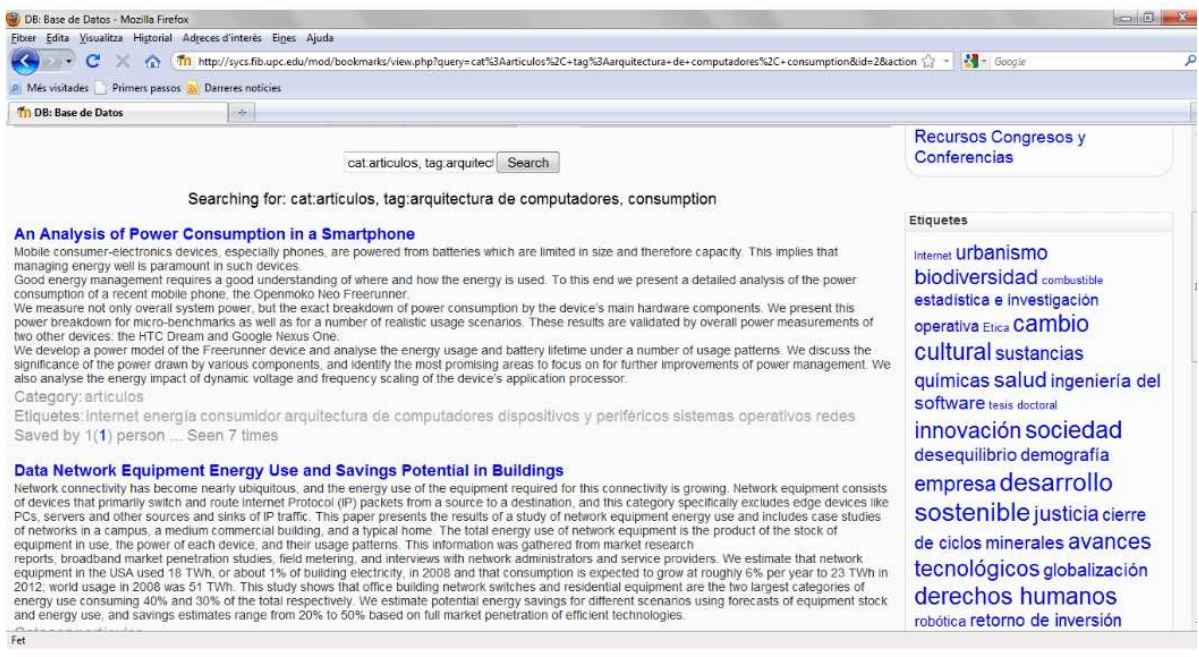

Figure 3. Example of search results.

A more restrictive search can be done as well by combining labels and / or free text, so that the search will be among the entries that meet all requirements. To make this type of search each parameter must be separated with a comma. For example, the search: "tag: solidarity, UN" (or "UN, tag: solidarity") will show the entries tagged with solidarity and that contain the word $U N$ in the title or description texts. Another example: the search "tag: solidarity, tag: innovation" will show the entries tagged with solidarity and innovation simultaneously. In this way, any searches can be refined as desired.

Usually, the tool is accessed as a guest user profile. This profile is assigned by default, it does not require any identification and allows the user to perform any type of search. However, there is also an editor user profile, which requires identification, but can also add new entries or modify the existing ones. In order to have an editor profile, it has to be requested, and it is only required to be an academic in the area of 
ICT or to have an interest and professional background related to the project. The authorization is currently managed by the project team.

Of course, after the initial release of the tool, it is in constant evolution. Current efforts are focusing on:

- Improving the search capabilities, allowing the realization of specific searches made by algebraic combinations of labels and free text (for example, list the entries with a label or another, but in any case, with some free text),

- Providing a more graphical, less text-oriented, user interface.

- Improving the graphic design.

\section{Final Thoughts and Conclusions}

Introducing professional skills in University studies is not an easy task. We can find teachers who deny its importance, teachers who do not know how to start, or teachers with some knowledge of the skills, but inadequately trained in it.

In all these cases, the most important thing to succeed in introducing these skills in the new degrees is information. It is important for teachers to have articles, books, websites and other sources of information to explain the importance of each competency. Knowing good practices and examples is essential to incorporate correctly the development and evaluation of this skill in the curricula.

The professional skill "Sustainability and Social Commitment" (SSC) is particularly complex, since many teachers have experience in other skills such as "teamwork" or "communication", but there is great ignorance in regard to SSC. In this paper we have presented a Knowledge Base aimed at helping teachers relate their Engineering course with SSC concepts.

A tool of this style is very important for success in incorporating this skill to a specific subject. The tool presented is universal, so that all interested teachers can consult it. In addition, teachers may request access particularly interested as editors to help expand its content (write access is not universal to prevent degradation of the information).

Finally, we want to add that some colleagues who have seen the tool and the process of generating labels have asked us to use (and modify) the tool to implement this skill in other areas of knowledge (such as Urban Planning, for example). Similarly, the concepts used and the tool itself can be used for work other professional skills.

\section{Acknowledgements}

This project has been partially funded by the UPC -STEP project, the Learning Apps project funded by Spanish Ministry of Science and Innovation in the program INNPACTO, and the project miPLE Spanish Ministry of Science and Innovation within fundamental research TIN program. 


\section{References}

1. Cobb, C.L., A. M. Agogino, S. L. Beckman and L. Speer. "Enabling and Characterizing Twenty-First Century Skills in New Product Development Teams", International Journal of Engineering Education, 24(2), 2008, pp. 420-433.

2. Colby, A. and W. M. Sullivan. "Ethics Teaching in Undergraduate Engineering Education". Journal of Engineering Education, 97(3), 2008, pp. 327-338.

3. Coyle, E.J., Jamieson, L.H. and Oakes, W.C. "EPICS: Engineering Projects in Community Service". Int'l J. of Engin. Educ 21(1) 2005, pp. 139-150.

4. Felder, R.M. "Sermons for grumpy campers". Chemical Engineering Education, 41(3):183 - 184, 2007.

5. Gößling-Reisemann, S. and Von Gleich, A. "Training Engineers for Sustainability at the University of Bremen”. Int'1 J. of Engin. Educ. 23(2) 2007, pp. 301-308.

6. Huntzinger, D.N., Hutchins, M.J. Gierke J.S. and Sutherland, J.W. "Enabling sustainable thinking in undergraduate engineering education." Int'l J. of Engin. Educ. 23(2) 2007, pp. 218-230.

7. López, D., F. Sánchez, J-L. Cruz and A. Fernández. "Developing Non-technical Skills in a Technical Course", Proceedings of the 37th Frontiers in Education Conference, pp F3B5F3B10. Milwaukee, WI, USA, October 2007.

8. McLaughlan, R.G. "Instructional Strategies to Educate for Sustainability in Technology Assessment". Int'l J. of Engin. Educ. 23(2) 2007, pp. 201-208.

9. National Academy of Engineering, "The Engineer of 2020. Visions of Engineering in the New Century", National Academy Press, 2004.

10. Shuman L.J., M. Besterfield-Sacre and J. McGourty. "The ABET Professional Skills-Can They Be Taught? Can They Be Assessed?” Journal of Engineering Education, 94(1), 2005, pp. 41-55.

11. Siller. T.J. "Sustainability and critical thinking in civil engineering curriculum". Journal of Professional Issues in Engineering Education and Practice, 127(3), 2001, pp. 104-108.

12. Tam, E. "Developing a Sustainability Course for Graduate Engineering Students and Professionals". Int'1 J. of Engin. Educ. 23(6) 2007, pp. 1133-1140.

13. Alier et at. "Interoperability for LMS: the missing piece to become the common place for elearning innovation" International Journal of Knowledge and Learning 2010 - Vol. 6, No. $2 / 3$ pp. $130-141$ 